\title{
Permeabilization surgery of the upper respiratory tract and its effects on sleep fragmentation and REM sleep
}

\author{
Ionut Tanase ${ }^{1,2}$, Claudiu Manea ${ }^{1,2,3}$, Codrut Sarafoleanu ${ }^{1,2,3}$ \\ ${ }^{1}$ ENT\&HNS Department, "Sfanta Maria" Hospital, Bucharest, Romania \\ ${ }^{2}$ CESITO Centre, "Sfanta Maria” Hospital, Bucharest, Romania \\ 3"Carol Davila" University of Medicine and Pharmacy, Bucharest, Romania
}

\section{ABSTRACT}

Usually, patients with sleep disorders may complain of tiredness, fatigue, daytime sleepiness, difficulty in concentrating, and can reach up to falling asleep in inappropriate situations - condition known as the Pickwick syndrome. To avoid these unpleasant symptoms, a series of surgical procedures regarding the anatomical structures involved in sleep apnea were developed. The article is a general review regarding the sleep disorders and the influence of upper airways permeability on the quality of sleep and the sleep staging distribution. Also, we present some preliminary data obtained in a clinical study underwent in CESITO Centre "Sfanta Maria" Hospital, Bucharest, involving patients with sleep pathology that had polysomnographic evaluations before and after various surgical procedures of nasal and pharyngeal permeabilization.

AIMS. To determine that permeabilization surgery of the upper airway tract may be used successfully in order to decrease the sleep fragmentation and increase the time of slow-wave sleep.

CONCLUSION. 6 months after the permeabilization surgery of the upper airway tract, the polysomnography reveals that the arousals index decreased and the sleep architecture undergoes changes that consist in decreasing the Stage 1 and Stage 2 sleep, therefore REM sleep reaches a better score.

KEYWORDS: sleep apnea, polysomnography, REM sleep, sleep fragmentation

\section{INTRODUCTION}

Sleep is represented by a reversible condition of disengagement from the environment and consists of complex physiological and behavioural processes ${ }^{1}$. Human's sleep includes two states - rapid eye movement (REM) sleep and non-rapid eye movement (NREM) sleep, which are chained gradually over night ${ }^{1}$.

Typical and synchronous cortical electroencephalogram (EEG) activity is found on EEG - polysomnographic investigation characterized by sleep spindles, $\mathrm{K}$ complex and slow waves associated with low muscle and minimal psychological activity tonus. In contrast, the REM sleep EEG is desynchronized and muscle reaction can occur mainly in dreams. The NREM sleep is divided in 3 stages (stages 1, 2 and 3$)^{2}$.

From the American Academy of Sleep Medicine (AASM) study we withhold that sleep stages have a cer- tain pattern: the REM sleep appears over a period of NREM sleep of approximately 80-100 minutes and it repeats over the night for about 4-5 episodes. The total duration of REM sleep must cumulate approximately 90 minutes per night ${ }^{3}$. The latter part of the night distribution of the REM sleep is thought to be influenced especially by the oscillation of the body temperature ${ }^{3,4}$. So, the sleep architecture is distributed as follows: sleep starts with a NREM episode; from one REM episode to another, it usually takes about 90 minutes of NREM sleep; REM sleep appeared primarily in the last third part of the night. According to the American Academy of Sleep Medicine, the percentage terms of sleep is represented as follows: Wakefulness - less that $7 \%$; NREM Stage 1 - 4-8\%; NREM Stage 2 - 47-58\%; NREM Stage 3 - 5-10\%; REM sleep - between 20 and $25 \%$.

The length of nocturnal sleep is directly influenced by a various number of factors such as age, sleep dep- 
rivation over last nights, heartbeat, the environment temperature, drugs, alcohol, and breath disorders such as apnea ${ }^{3,4}$.

Sleep disorders cause not only sleep fragmentation and poor quality of sleep, but also daytime sleepiness and other pathologies like hypertension and increased risk of vascular disease ${ }^{4}$.

One of the most important factors that influence the sleep architecture is age. Probably the most eloquent example is represented by the sleep transition in the first year of life, when from the weakness stage the new-born infants enter directly into the REM sleep, phenomena known as active sleep ${ }^{5}$.

Sleep loss leads to a deeper and longer recovery of REM sleep. On polysomnographic investigation, EEG shows preponderant slow waves because NREM sleep tends to be preferentially recovered compared with REM sleep, which tends to recover only after the recuperation of slow-wave sleep ${ }^{6}$.

The fluctuation of the environment temperature tends to disrupt sleep architecture, and it seems that REM sleep is more sensitive than the superficial sleep stages with slow waves. Parmeggiani et al. report that the human body has a minimal ability to thermoregulate particularly during the REM sleep. Knowing this, the reaction of the body's thermoregulation by sweating or shivering is a condition that helps the patient to remain in NREM sleep type instead of reaching the REM sleep ${ }^{7}$.

Many common drugs tend to affect the sleep distribution and, for example, benzodiazepines tend to suppress slow-wave sleep and have no consistent effect on REM sleep. Van Reen E et al. demonstrated that tricyclic antidepressants and certain selective serotonin reuptake inhibitors tend to suppress REM sleep8.

High alcohol consumption before nocturnal sleep favours the appearance of slow-wave sleep (SWS). So, in the second half of the night, after alcohol has been metabolised, the REM sleep occurs ${ }^{9}$.

Also, marijuana consumption can affect the sleep distribution by reducing the REM sleep ${ }^{9}$.

One of the most important factors that are involved in sleep apnea and therefore in sleep fragmentation is represented by the anatomical traits of the organ involved in the appearance of the apnea. Further, we will focus on the following symptoms and also the anatomical outlines involved:

- Poor muscle tonus - The snoring and the sleep apnea episodes appear when muscles are too relaxed and the tongue falls backwards into the airway or the throat muscles draw in from the sides into the airway. This is a natural condition during deep sleep, but it can be aggravated by alcohol and drug consumption ${ }^{10}$.

- Excessive bulkiness of upper airway calibre. For example, children with large tonsils and ade- noids often snore and may also present sleep apnea. Overweight people may have excess soft tissue around the neck, condition that can lead to airway narrowing and consecutively to OSA. A rare cause of obstruction is represented also by nasal tumors with nasopharynx extension or rhinopharynx cysts ${ }^{11}$.

- Long soft palate and/or uvula. Maybe the most common pathology incriminated for OSA is represented by the large size of this anatomic element. An enlarged soft palate can have a narrowing effect on the oropharynx. At the same time, the excessive length of the soft palate and/or of the uvula acts as a noisy flutter valve during relaxed breathing. ${ }^{12}$.

- Obstructed nasal airways. Although it seems that nasal obstruction alone does not cause sleep apnea, due to the extra effort required to pull air through it, nasal pathology creates an exaggerated vacuum in the throat that pulls together hypotonic tissues of the throat, and snoring and obstructive apnea appear ${ }^{13}$.

\section{UPPER AIRWAY PERMEABILIZATION SURGERY - BRIEF LITERATURE REVIEW}

One of the current challenges of the ENT doctor is represented by the obstructive sleep apnea surgery. In the literature, we found several techniques dedicated to the correction of OSA with variable success rates. The correction of an obstructed nasal airway is considered an important component in obstructive sleep apnea OSA treatment ${ }^{14-16}$. Even if there are several clinical reports and experimental data documenting the existence of nasal obstruction-induced OSA, there are a lot of controversies regarding the role of improved nasal breathing in treating OSA ${ }^{14,16-18}$. The exact role of nasal obstruction in obstructive sleep apnea is unclear. One explanation can be represented by the reduced nasal cross sectional area which causes an increased nasal resistance and favours the inspiratory collapse of the oropharynx, hypopharynx, or both $^{19,20}$.

Olsen and Kern ${ }^{17}$ had demonstrated in their study the importance of the nasal cycle and nasopulmonary reflex in maintaining adequate blood oxygenation, thus sustaining the role of nasal breathing in apnea episodes. After nasal permeabilization surgery, the lowest level of SpO2 increased significantly.

In the literature we found no results to contradict the necessity of correction of an obstructed nasal airway in the surgical management of OSA. One of the most significant benefits of nasal surgery is represented by the decrease in the appearances of arous$a^{14}{ }^{14-16}$. On the other hand, in patients diagnosed with 
severe apnea, nasal surgery proved to decrease the CPAP titration levels that improved compliance of CPAP therapy and reduced morbidity and mortality of $\mathrm{OSA}^{16}$. Many studies recommend nasal surgery in order to improve compliance with treatment using nasal continuous positive airway pressure (CPAP) / bilevel positive airway pressure (BiPAP) devices in patients with obstructive sleep apnea (OSA) ${ }^{13-15}$. At the same time, the impact of nasal surgery on objective measurements in OSA patients is unclear, an important number of authors sustaining in their studies the limitation of this surgery in treating sleep apnea ${ }^{15-18}$.

Jiten et al. concludes that obstructive sleep apnea syndrome is a disease process that results in pharyngeal narrowing or complete collapse while asleep and this is the main cause for developing sleep fragmentation, frequent arousals, abnormally negative intrathoracic pressures, and daytime symptoms of fatigue and cognitive impairment ${ }^{21}$.

Besides the nasal obstruction, one of the most important factors involved in sleep disorders is represented by the soft palate flaccidity and also the tonsillar pathological modifications. In order to prevent sleep apnea caused by anatomical variations of the soft palace, many techniques were developed. From those we mention Laser Assisted uvulopalatoplasty, a surgical technique developed by Kamamy ${ }^{22}$ in order to treat snoring and also sleep apnea. It's an offshoot from uvulopharyngopalatoplasty (UPPP) and it consists in a staged resection of the uvula and soft palate under local anesthesia (vertical palatal incisions lateral to the uvula followed by subtotal uvulectomy).

Mickelson $^{23}$ et al. report that, after LAUP surgery, better postoperative outcomes were obtained, doubled by polysomnographic results. 6 weeks postoperatively, the apnea-hypopnea index (AHI) decreased from 19 events an hour to 4 events an hour, the respiratory disturbance index (RDI) decreased from 31 to 16 / hour.

Laser assisted uvulopalatoplasty is not routinely recommended as a treatment for patients with obstructive apnea syndrome because LAUP does not generally normalize the apnea-hypopnea index (AHI) in particular in patients with severe OSA. In order to demonstrate this, the literature bares a lot of studies that did not show improvement in secondary outcomes on polysomnographic investigation. Some studies actually saw worsening of the overall $\mathrm{AHI}^{24-26}$.

Ryan and Love developed a study on 44 patients that underwent LAUP surgery and the polysomnographic results review an important decrease in AHI with an average of 27 percent 6 months postoperatively ${ }^{27}$.

In literature we also found a series of side effects reported after this type of surgery - for example, Carenfelt reported that, from a total of 100 patients, 2 developed velopharyngeal insufficiency ${ }^{28}$.
All these types of surgery have in common some goals that are likely to decrease the snoring and apnea episodes. By decreasing those events in the background, we will meet fewer arousal episodes and, as a result, a better layout of sleep stages distribution and secondarily a better REM sleep percent.

\section{OUR CONSIDERATIONS}

In November 2015, a Center of Excellence for Research of Sensorial and Sensitive Disorders, Study of Infecto-inflammatory, Tumoral and Obstructive Aerodigestive Pathology (CESITO) was founded in the ENT\&HNS Clinic of "Sfanta Maria" Hospital, in Bucharest. The Sleep Disorders Laboratory of "CESITO" Center was fully equipped with Alice 6 LDX polysomnograph.

We decided to investigate the influence of the permeabilization surgery of the upper airway upon sleep distribution. At the present moment, we are undergoing a prospective study, started in October 2015, our goal being to demonstrate that after this particular type of surgery has been performed, the prevalence of arousals will decrease and the REM sleep will be easily reached. In order to show this, we will present two cases of patients diagnosed with sleep apnea on polysomnographic investigation. We report the result of the first investigation and also the outcomes at six months after surgery.

We mention that the patients with severe obstructive sleep apnea $(\mathrm{AHI}>30 / \mathrm{h})$ are strongly encouraged to pursue CPAP therapy because of its proven safety and efficiency ${ }^{29,30}$.

All the patients admitted to the ENT Department of "Sfanta Maria" Hospital with sleep disorders are evaluated and the following exclusion and inclusion criteria in the study are used:

- Inclusion criteria are represented by: patients with sleep apnea syndrome with different causes of nasal obstruction such as nasal septum deviation, hypertrophic chronic rhinitis; pharynx pathology (long soft palate and/or uvula, large tonsils, etc.).

- Exclusion criteria: massive facial malformations such as retrognathia, patients with apnea of pulmonary or neurological origin, patients diagnosed with severe apnea whose correct indication is non-invasive ventilation at home.

Before recruitment, each eligible patient undergoes a careful otorhinolaryngological evaluation. The ENT examination follows some of the potential changes on the normal anatomy of the nasal cavity, the oral cavity, the oropharynx or hypopharynx like poor tonus of the pharynx muscular layer, excessive bulkiness of the upper airway calibre, long soft palate or long and large 
uvula, obstructed nasal airways of different causes like deviated nasal septum, hypertrophic inferior turbinates.

For each of this pathology condition, different types of surgery procedures are used.

All patients that qualify for this study should perform a polysomnographic examination both preoperatively and 6 and 12 months postoperatively, in order to document the effect of ENT surgery on sleep fragmentation and REM sleep distribution.

The following parameters are evaluated during this study: sleep distribution (NREM stages N1, N2, N3 and REM sleep), snoring characteristics (snoring episodes and total snoring time), oxygen saturation, total apnea number (obstructive apnea, central apnea, mixed apnea), hypopnea, apnea-hypopnea index (AHI), maximum duration of apnea and/or hypopnea, arousals total number, arousals index. Also, the daytime sleepiness is subjectively evaluated with the Epworth questionnaire before the first polysomnographic investigation and then at 6 months postoperatively.

\section{POLYSOMNOGRAPHY (PSG)}

Standard electroencephalographic (EEG), electrooculographic (EOG), and electromyographic (EMG) criteria where used to document various sleep stages. According to the AASM, 16 EEG was recorded with electrodes applied at C3-A2 and C4-A1 and also the EMG activity was recorded from the submental muscles and anterior tibialis muscles ${ }^{29}$. We used a full equipped system of Alice 6 LDX to study and diagnose sleep disorders.

Apneas are defined as cessation of airflow in the nose and mouth for longer than 10 seconds and were documented by an infrared $\mathrm{CO} 2$ analyser which records from both the nose and the mouth and also with an pulse oximeter sensor attached to the index finger that records the level of oxygen saturation ${ }^{31,32}$.

Hypopneas are defined as reductions of more than $50 \%$ in the excursion of the sum channel of a respiratory inductance plethysmography measuring chest wall and abdominal movement $\mathrm{t}^{31,32}$.

Cardiac activity is monitored with a single electrocardiographic (ECG) lead (modified V2).

We manually score the complete record night investigation for sleep stage and apnea type and duration. The severity of sleep apnea was expressed by an index of apnea and hypopnea over one hour of investigation (AHI). Also, on EEG activity, we monitored the prevalence of arousals.

For the snoring flags, we use a microphone placed on the laryngeal region for recording of tracheal sounds audible. Moreover, in this investigation, the following indicators are monitored: respiratory effort - chest-wall and abdominal movement via strain gauges, piezoelectric belts, inductive plethysmography, impedance or inductance pneumography, endoesophageal pressure, or by intercostal EMG; nasal and/or oral airflow - thermistor or pneumotachograph; body position - mercury switches or by direct observation; limb movements (arms and legs) - EMG (Figure 1).

We consider that all patients with OSA should be assessed with PSG both before and after surgery, because subjective analysis often does not accurately correlate with objective changes in OSA.

\section{QUALITY OF LIFE AND SLEEPINESS}

The Epworth and Calgary Sleep Apnea Quality of Life Index were administrated to all the patients admitted in our clinic for this pathology before and after surgery ${ }^{33}$.

\section{CASE REPORT}

\section{Case 1}

A 40-year-old man, worker in shifts, obese class I $(180 \mathrm{~cm}, 104 \mathrm{~kg}$, body mass index (BMI) of 32.1$)$, referred to our clinic complaining of sleep apnea and snoring both reported by entourage. The patient also reported progressive decreased ability to work, bilateral nasal obstruction, anterior serous rhinorrhea. The Epworth Scale revealed daytime sleepiness with a score of 12.

The ENT evaluation revealed: hypertrophy of the inferior turbinates (significant volume reduction after nasal decongestion), nasal septum deviation on the left side but with no obstructive effect grade I; long

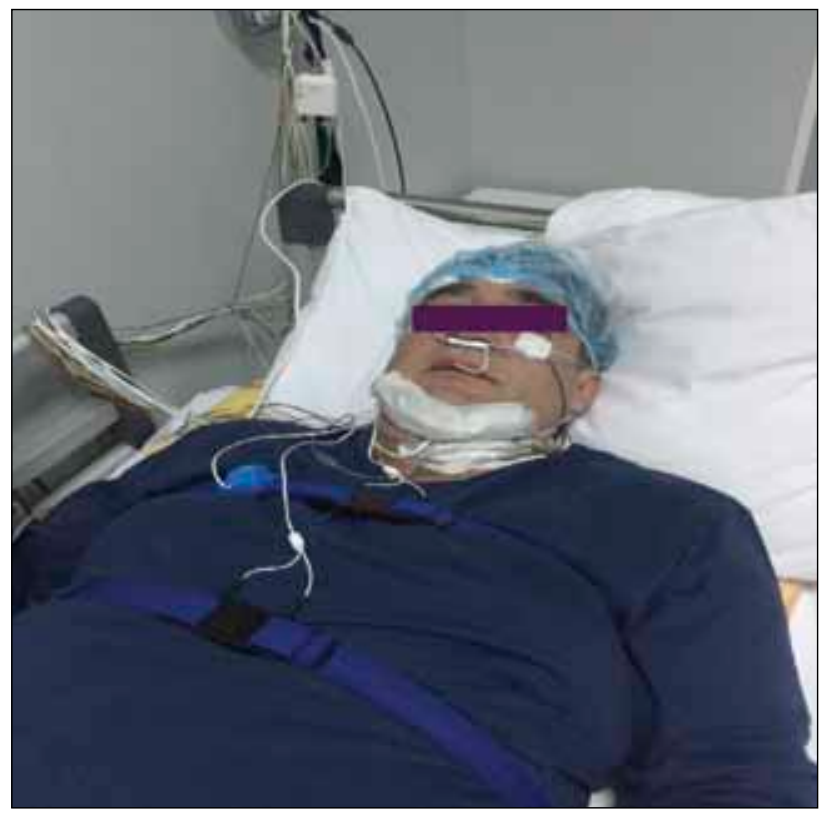

Figure 1 Polysomnography electrodes placement. 
soft palate and large uvula, with normal aspects of the tonsils and of both hypopharynx and larynx; Class III Mallampati score.

The preoperative polysomnography highlighted a moderate sleep apnea $(\mathrm{AHI}=18.2 / \mathrm{h})$, with a total REM sleep of 63.2 minutes, sleep efficiency of $69.5 \%$ from a total of 530 minutes Sleep Period Time and 393 minute Total Sleep Time, 397 arousals, SpO2 medium $95 \%$ and SpO2 minimum $84 \%$. A total of 120 obstructive sleep events were recorded, from which 57 were obstructive apneas, 8 central apneas, 4 mixed apneas, and 51 hypopnea events. The maximum duration of an obstructive event was 50.5 seconds. The snoring episodes number was 88 with a total duration of 28.1 minutes.

Laser assisted uvulopalatoplasty (LAUP) was performed under local anaesthesia without any premedication. $10 \%$ Lidocaine spray was applied to the soft palate followed by infiltration of either side of the base of the uvula and soft palace area with mixture of Xylene $2 \%$ and adrenaline. We use the diodes laser power at $15 \mathrm{~W}$ in continuous mode and also Radiofrequency is used in order to produce thermal lesions within the tissues for stiffening the neo-soft palate.

After LAUP surgery, 6 months postoperatively, we observed an important decrease in the apnea and hypopnea index (AHI) from a total of 120 to 85 events (Figure 2). After surgery, most of the events were hypopneas (52 hypopneas and 27 obstructive apneas) compared to the first examination when the obstructive apnea was prevalent ( 57 obstructive apneas and 51 hypopneas). We saw better results also in oxygen blood saturation levels (SpO2 reached a minimum of $88 \%$, so it was improved by 4 percents compared to the first examination when SpO2 was 84\%) (Figure 3). The maximum duration of an obstructive respiratory event decreased from approximately 50 seconds to 20 seconds. The snoring episodes decreased from 88 to 47 for a cumulated total snoring time of 28.1 minutes during the first examination to only 15 minutes.

The postoperative daytime sleepiness index on the Epworth questionnaire was improved from 12 to 8.

\begin{tabular}{|c|c|c|c|c|c|c|c|c|c|c|c|c|c|c|c|c|c|}
\hline & $\mathrm{CA}$ & 89 & Ma & Apes: & $1602 x^{2}$ & $A+11$ & 다제 & Total & 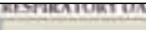 & & & & & & & & \\
\hline Alaber: & 5 & 87 & i & 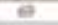 & 31 & 120 & 0 & (120) & & CA & 93 & $\mathrm{Ma}$ & Apes: & Itrox' & $A=B$ & WIXA & Intat \\
\hline \multirow{2}{*}{ Mran Denestiont } & \multirow{2}{*}{186} & \multirow{2}{*}{ rer } & \multirow{2}{*}{14} & \multirow{2}{*}{175} & \multirow{2}{*}{26} & \multirow{2}{*}{22} & \multirow{2}{*}{ wa } & \multirow[t]{2}{*}{$x 2$} & Numbler & 4 & 2 & 2 & 3 & \pm & Es & 0 & (5) \\
\hline & & & & & & & & & Mesas Dur thect & D24 & E3 & 1eas & 167 & $2 x$ & 293 & eo & 10 \\
\hline Mas Dar thed: & 160 & we & \multirow[t]{2}{*}{$m e$} & \multirow[t]{2}{*}{$x \in 0$} & es & & \multirow[t]{2}{*}{ at } & \multirow[t]{2}{*}{ ces } & Men Drations & & & & & & & & \\
\hline \multirow{2}{*}{ Tolal Den finint } & \multirow{2}{*}{18} & \multirow{2}{*}{ 12? } & & & \multirow{2}{*}{201} & \multirow{2}{*}{63} & & & & & & & & & & w & We \\
\hline & & & 10 & 24 & & & we & 25 & Toul Dor teine: & as & zs & As & 12 & 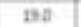 & as: & BQD & $\mathrm{xu}$ \\
\hline \multirow[t]{2}{*}{7 at ISI: } & \multirow[t]{2}{*}{ as } & \multirow{2}{*}{45} & \multirow[t]{2}{*}{ as } & \multirow[t]{2}{*}{12} & \multirow[t]{2}{*}{31} & 123 & 06 & 10.) & & & & & & & & & \\
\hline & & & & & & & & & Seat TSt & 22 & 13 & 21 & 23 & 67 & 200 & 60 & 70 \\
\hline 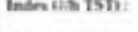 & 12 & W & Us & 10.5 & 73 & 133 & ed & & Indes & 36 & $6 \mathbf{f}^{2}$ & (3) & 47 & & & & \\
\hline ETMCanet: & & & & & & & & th & & & & & & & $22 \pi$ & $\theta$ & \\
\hline & 2 & 2 & 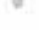 & 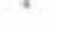 & $\pi$ & in & 0 & a & HuCent: & $\mathrm{I}$ & $\mathrm{x}$ & 8 & 2 & 3 & 7 & $\theta$ & 7 \\
\hline NaIMComit: & 4 & $\$ 8$ & 4 & 68 & N & 102 & $\theta$ & Ia & N⿴囗МС" & & & & & & & & \\
\hline EMMinde & & & & & & & & & 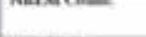 & 3 & $x$ & 2 & 31 & e & $\mathrm{n}$ & 0 & $\mathrm{n}$ \\
\hline 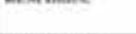 & 100 & Do & was & 15 & 47 & *6 & 0 & 16 & HrMlaks: & ex & 02 & ate & 4 & 35 & 49 & 00 & 69 \\
\hline NerM tales: & 13 & 123 & as & 166 & 3 & 2.0 & de & 23 & NatM lindex & at 6 & 19 & $\Delta 4$ & is & $\mathrm{Am}$ & 147 & 60 & 16 \\
\hline at & & & & & & $=$ & $\mathrm{t}$ & & & & & & & & & & \\
\hline
\end{tabular}

Figure 2 Before and after LAUP surgery ( 6 months postoperatively), the polysomnography investigation revealed that the total number of 120 obstructive events decreased to a total of 85 events and the most important improvement was recorded in the number of obstructive apneas that decreased from 57 to 27.

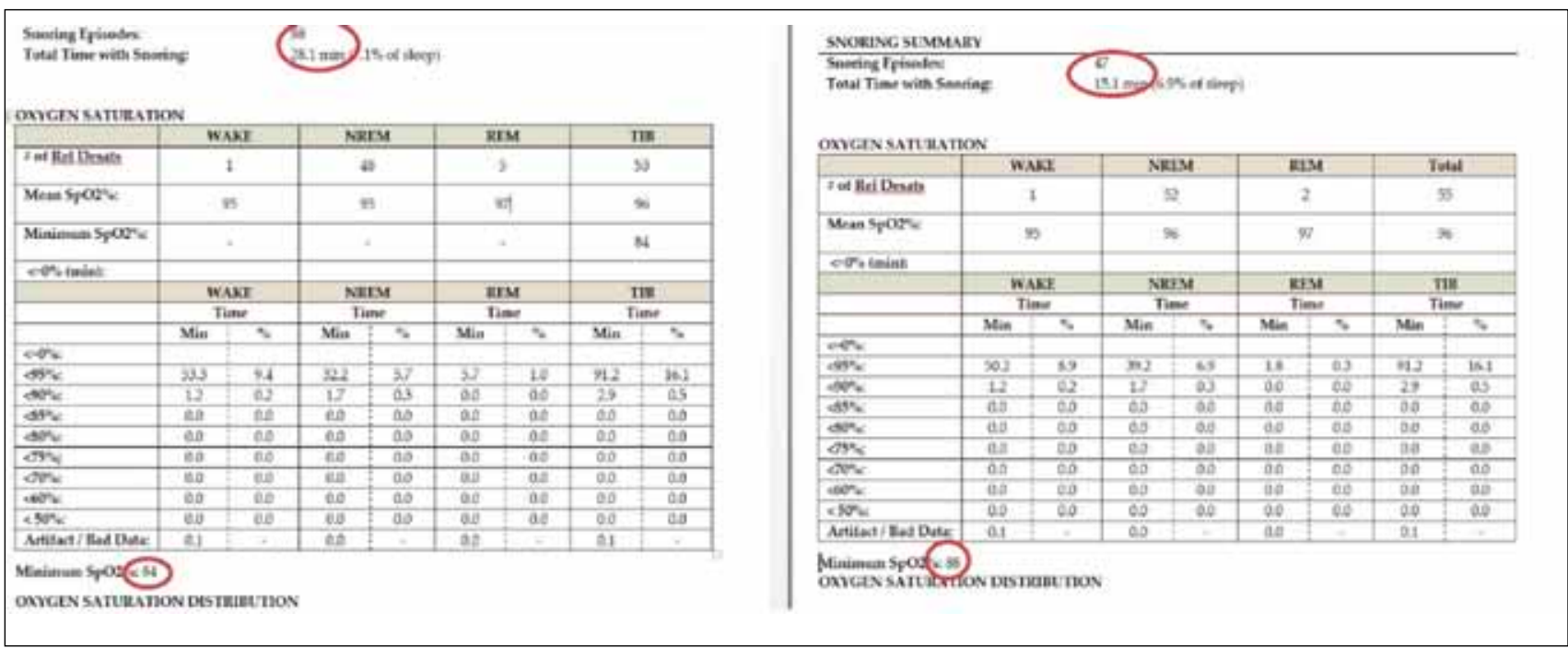

Figure 3 Before and after LAUP surgery ( 6 months postoperatively), the polysomnography investigation revealed that the minimum SpO2 improved with 4 percents from 84 to $88 \%$ and also the snoring episodes decreased from 88 to 47 events. 
The sleep architecture (Figure 4) also has suffered important modifications - N1 from 130.1 minutes $(33.2 \%)$ to 155 minutes (representing $38.2 \%$ from the total sleep time), N2 from 157.5 minutes $(34.3 \%)$ to 157.5 minutes $(39 \%)$, N3 from 56.0 minutes $(15.5 \%)$ to 46 minutes $(14.5 \%)$ and REM sleep from a total 63.2 minutes $(17 \%)$ to 85.5 minutes $(21.2 \%)$. We mention that the total sleep recording time was approximately the same - 615 minutes preoperatively and 630 minutes postoperatively - and the total sleep time is similar in size - 393.5 minutes compared to 404 minutes from the second investigation.

Arousals index decreased from $60.5 / \mathrm{h}$ to $37.5 / \mathrm{h}$, from a total number of events of 397 to 199 (Figure 5).

6 months postoperatively, the body mass index improved from 32.1 to 29.6 (from obesity grade I to overweight) and this can be one of the most important factors implicated in the modifications of the polysom- nography values. Another reason that can be involved in the differences between those two examination results can be the "first night effect" 34 . Masako et al. demonstrated that on the first night polysomnographic examination, alpha waves activity increased and the activity of the wake-promoting system during the sleeponset period was improved ${ }^{34}$. Other studies ${ }^{35,36}$ revealed significant differences in the same patients' evaluations between two consecutive nights, as reflected by the scores obtained on the Groningen Sleep Quality Scale.

\section{Case 2}

A 56-year-old man referred to our clinic complaining of sleep apnea and snoring, both reported by entourage, nasal obstruction on both nostrils, and daytime sleepiness (Epworth score $=13$ ). Body mass index was 33.2 (height $170 \mathrm{~cm}$, weight $96 \mathrm{~kg}$ ) - obese grade I.

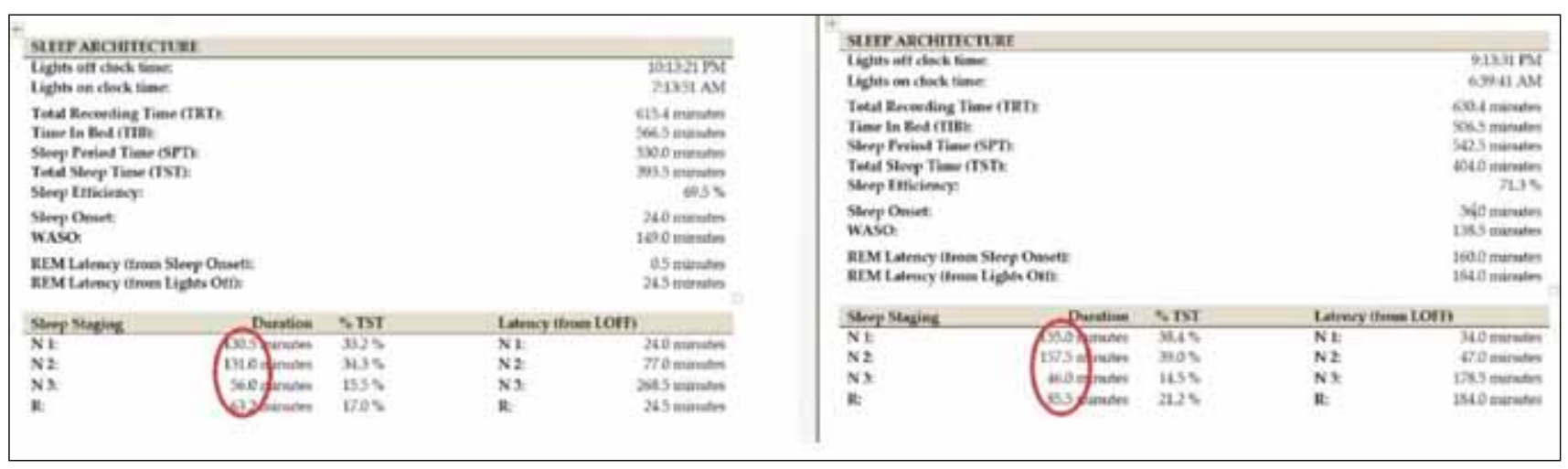

Figure 4 Before and after LAUP surgery ( 6 months postoperatively), the polysomnography investigation revealed that the Sleep Stages were different. REM sleep increased from 63.2 minutes to 85.5 minutes.

\begin{tabular}{|c|c|c|c|c|c|c|}
\hline \multicolumn{7}{|l|}{ AROUSALS } \\
\hline & REM & NREM & Arousals & Awakenings & $\mathrm{Ar}+\mathrm{Aw}$ & $\begin{array}{c}\mathrm{Ar}+\mathrm{Aw} \\
\text { Index }\end{array}$ \\
\hline Respiratory: & 11 & 17 & 28 & 28 & 56 & 8.5 \\
\hline Leg Movement: & 60 & 44 & 104 & 37 & 141 & 21.5 \\
\hline Snore: & 0 & 3 & 3 & 18 & 21 & 3.2 \\
\hline Spontaneous: & 43 & 39 & 82 & 97 & 179 & 27.3 \\
\hline Total: & 114 & 103 & 217 & 180 & 397 & 60.5 \\
\hline Arousal Index: & 54.3 & 23.1 & 33.1 & 27.4 & 60.5 & - \\
\hline \multicolumn{7}{|l|}{ AROUSALS } \\
\hline & REM & NREM & Arousals & Awakenings & $\mathrm{Ar}+\mathrm{Aw}$ & $\begin{array}{c}\mathrm{Ar}+\mathrm{Aw} \\
\text { Index }\end{array}$ \\
\hline Respiratory: & 9 & 116 & 127 & 27 & 154 & 29.1 \\
\hline Leg Movement: & 0 & 12 & 14 & 2 & 16 & 3.0 \\
\hline Snore: & 0 & 7 & 8 & 0 & 8 & 1.5 \\
\hline Spontaneous: & 0 & 16 & 19 & 2 & 21 & 4.0 \\
\hline Total: & 9 & 151 & 168 & 31 & 199 & 37.5 \\
\hline Arousal Index: & 54.0 & 29.4 & 31.7 & 5.8 & 37.5 & - \\
\hline
\end{tabular}

Figure 5 Before and after LAUP surgery ( 6 months postoperatively), the polysomnography investigation revealed that the arousals index decreased from a total $60.5 / \mathrm{h}$ to a better score of 37.5 events per hour. 
The ENT examination revealed: right obstructive nasal septal deviation grade III, hypertrophy of the inferior turbinates (no volume reduction after nasal decongestion), normal aspect of the uvula and soft palate, hypertrophy of both tonsils; Mallampati score II.

The preoperative polysomnography revealed a moderate sleep apnea syndrome $(\mathrm{IAH}=21.4 / \mathrm{h})$ with 149 total obstructive events, from which 41 obstructive events, 108 hypopneas. The maximum duration of a sleep event was 57.5 seconds. The scoring episodes number was 94 with a total duration of 20.1 minutes. The total REM sleep time was 40.5 minutes, sleep efficiency of $56.5 \%$ from a total of 508 minutes Sleep Period Time and 334.5 minute Total Sleep Time, 397 arousals, $\mathrm{SpO} 2$ medium 94 and $\mathrm{SpO} 2$ minimum 84.

To resolve the obstructive pathology, under general anaesthesia with endotracheal intubation, septoplasty and inferior turbinates mucotomy were performed. Nasal packing was removed on the first postoperative day, and routine postoperative saline nasal irrigation and debridement were performed.

6 months after the nasal permeabilization surgery, an important decrease in apnea and hypopnea index (AHI) can be observed - from a total of 149 to 98 events (Figure 6). 6 months after the nasal surgery, most of the events were still hypopneas (73 hypopneas and 25 obstructive apneas) compared to the first examination (41 obstructive apneas and 108 hypopneas). Better results in the level of oxygen blood saturation were recorded - SpO2 reached a minimum of $84 \%$, so it was improved by 14 percents compared to the first examination when SpO2 was $70 \%$ (Figure 7). The maximum duration of an obstructive respiratory event increased from approximately 57.5 seconds to $59 \mathrm{sec}-$

\begin{tabular}{|c|c|c|c|c|c|c|c|c|}
\hline & $\mathrm{CA}$ & OA & MA & Aprea & Hoges' & $A+H$ & RERA & Total \\
\hline Neniber: & 0 & (i1) & 0 & il & 105 & 10 & 0 & (19) \\
\hline Mean Der isect: & 0.0 & 145 & 0.0 & 14.5 & 20.3 & 18.7 & 0.0 & 18.7 \\
\hline Max Dur iseck: & 0.0 & 250 & 00 & 250 & 575 & & 00 & 575 \\
\hline Total Dur (mia): & 00 & 99 & 00 & 99 & 365 & $\$ 6.4$ & 00 & that \\
\hline Sof TST: & 0.0 & 24 & 00 & 24 & 57 & 114 & 00 & 11.1 \\
\hline Indesith TST): & 0.0 & 5.9 & 0.0 & is & 155 & 214 & 0.0 & \\
\hline REM Count: & 0 & 23 & 0 & 23 & 99 & $n$ & 0 & 72 \\
\hline NREM Cesent: & 0 & 15 & 0 & is & 97 & 7 & 0 & 7 \\
\hline REM Indec: & 0.0 & 15.1 & 0.0 & 15.1 & 32.1 & 472 & 00 & 672 \\
\hline NREM Indec & 0.0 & 33 & 0.0 & 33 & 10.9 & 142 & 0.0 & 142 \\
\hline
\end{tabular}

\begin{tabular}{|c|c|c|c|c|c|c|c|c|}
\hline & CA & OA & MA & Apnea & Hyres: & $\mathrm{A}+\mathrm{H}$ & IEERA & Total \\
\hline Nuanber: & 0 & (b) & 0 & 25 & 735 & 98 & 0 & (c) \\
\hline Mean Dur iseck & 0.0 & 209 & 20 & 209 & 242 & 234 & 0.0 & 23.4 \\
\hline Max Dur isect: & 00 & 420 & 0.0 & 420 & 990 & & 00 & 59.0 \\
\hline Total Dux (nind: & 00 & 37 & $Q 0$ & 8.7 & 295 & 382 & 00 & 362 \\
\hline sot TSI: & 00 & 26 & 0,0 & 26 & 8.5 & 11.4 & 0.0 & 114 \\
\hline Index $(2 \mathrm{~h}$ TST: & 0.0 & 45 & 0,0 & 15 & 13.1 & 17.6 & 00 & \\
\hline REM Count: & 0 & 1 & 0 & 1 & 6 & 7 & 0 & 7 \\
\hline NREM Count: & 0 & 24 & 0 & $x$ & 67 & 91 & 0 & 91 \\
\hline REM Index & 00 & 15 & 0.0 & 15 & 89 & 10.4 & 00 & 20.4 \\
\hline NREM Indec & 00 & 49 & 0.0 & 49 & 137 & 18.6 & 0.0 & 186 \\
\hline
\end{tabular}

Figure 6 After nasal repermeabilization by both septoplasty and mucotomy (6 months postoperatively), the total number of 149 obstructive events decreased to a total of 98 events and, also, an improvement was recorded in obstructive apneas (from 41 to 25 ), hypopneas (from 108 to 73 ), but the maximum duration of hypopnea it increased from 57.5 seconds to 59 seconds.

\begin{tabular}{|c|c|c|c|c|c|c|c|c|c|c|c|c|c|c|c|c|c|}
\hline \multicolumn{9}{|c|}{ SNOWINC SUMMENIKY } & \multicolumn{9}{|c|}{ SNORTVG STMMARY } \\
\hline \multirow{2}{*}{\multicolumn{9}{|c|}{$\begin{array}{l}\text { Snoring Tyivade: } \\
\text { Total Time with Snating }\end{array}$}} & \multirow{2}{*}{\multicolumn{9}{|c|}{$\begin{array}{l}\text { Shotins t pivdes: } \\
\text { Total Time with Sanving }\end{array}$}} \\
\hline & & & & & & & & & & & & & & & & & \\
\hline \multicolumn{9}{|c|}{ OMGENSATIRATION } & \multicolumn{9}{|c|}{ OXYGEN SATURA } \\
\hline & \multicolumn{2}{|c|}{ WAKE } & \multicolumn{2}{|c|}{ NEFM } & \multicolumn{2}{|c|}{ HIM } & \multicolumn{2}{|c|}{ IIII } & & \multicolumn{2}{|c|}{ WAKE } & \multicolumn{2}{|c|}{ NEEM } & \multicolumn{2}{|c|}{ IIM } & \multicolumn{2}{|c|}{ TIII } \\
\hline Fof Red Drust: & \multicolumn{2}{|c|}{1} & \multicolumn{2}{|c|}{ ist } & \multicolumn{2}{|c|}{30} & \multicolumn{2}{|c|}{314} & I of Rel Drowh & \multicolumn{2}{|c|}{1} & \multicolumn{2}{|c|}{ 4s } & \multicolumn{2}{|c|}{5} & \multicolumn{2}{|c|}{93} \\
\hline Mlem SpO2N & & & \multicolumn{2}{|c|}{92} & \multicolumn{2}{|c|}{92} & \multicolumn{2}{|c|}{ s } & Mean SpOzer & \multicolumn{2}{|c|}{ (x) } & \multicolumn{2}{|c|}{92} & \multicolumn{2}{|c|}{$\mathbf{w}$} & \multicolumn{2}{|c|}{56} \\
\hline Minimem SpOQV & & & & & & & & & Minimum SpOĚs & & & & & & & & \\
\hline entunias: & & & & & & & & & contiminis & & & & & & & & \\
\hline & & & & & & & & & & & & & & & & & \\
\hline & & & & & & & & & & & & & & & & & \\
\hline & Min & 5 & Min & 5 & Min & 5 & Min & 8 & & Min & 5 & Min & 5 & Min & 5 & Min & 5 \\
\hline eov & & & & & & & & & $\cos x$ & & & & & & & & \\
\hline $\cos 2$ & 70 & 33 & 28,7 & Why & 220 & 47 & 2853 & So. 2 & $\phi^{*}=$ & 383 & 94 & 32 & 39 & 57 & 10 & 92 & 16.1 \\
\hline$\infty=$ & is & 09 & HSA & 227 & 100 & 20 & 1003 & 2.6 & $60 \%$ & 12 & 02 & 15 & Q3 & 00 & 20 & 29 & es \\
\hline 830 & 02 & 0.9 & 366 & 72 & 43 & 09 & 41.6 & II & 690 & 0.0 & $O E$ & 00 & 00 & 00 & 00 & 00 & $\infty 0$ \\
\hline $\operatorname{sor} x$ & 00 & 00 & 70 & 14 & 0.5 & 0.2 & 73 & 15 & Son & 00 & 00 & 00 & 00 & 00 & 00 & 00 & 00 \\
\hline $28 \%$ & 00 & $a 0$ & 1.1 & 02 & 20 & 00 & 11 & 22 & ose & 00 & 00 & 0.0 & 00 & 00 & 00 & 00 & 00 \\
\hline 60te & 00 & 00 & 00 & $\infty$ & 20 & 00 & 0.0 & 00 & 200 & $\infty$ & 00 & $\infty$ & $\infty 0$ & 00 & 00 & 00 & 00 \\
\hline C60\% & 0.0 & 0.0 & 00 & 0.0 & 00 & 0.0 & 0.0 & 00 & ext & 00 & $\infty$ & $\omega$ & 0 & 00 & 00 & 00 & $\infty$ \\
\hline$\angle \operatorname{son} 6$ & 0.0 & $0 \theta$ & 00 & 0.0 & 00 & 00 & 0.0 & $\infty$ & $30=$ & 00 & ED & 00 & 00 & 00 & 20 & 00 & 00 \\
\hline 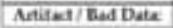 & 75 & 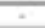 & 00 & 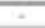 & 00 & - & 95 & - & Antisad/Bad Date & at &. & 0.0 & - & 00 & - & a) & - \\
\hline
\end{tabular}

Figure 7 The minimum level of SpO2 improved with 14 percents from 70 to $84 \%$ after septoplasty and mucotomy and the snoring episodes decreased with only 6 events ( 6 months postoperatively). 
onds. The snoring episodes decreased, but not significantly, from 94 to 88 ; however, they cumulated a total snoring time that increased from 20.1 minutes to 28.1 minutes.

Important changes occurred in the sleep architecture, such as: stage $\mathrm{N} 1$ from 101.0 minutes $(30.2 \%)$ to 124.5 minutes (representing $32.0 \%$ of the total sleep time), N2 from 131.5 (39.3\%) to 218.0 minutes $(52.2 \%)$, N3 from 61.5 minutes $(15.5 \%)$ to $3.5 \mathrm{~min}$ utes $(0.8 \%)$ and REM sleep from a total of $40.5 \mathrm{~min}$ utes $(12.1 \%)$ to $71.5 \%$ (14.9\%) (Figure 8$)$. We mentioned that the difference in the total sleeping recording time was important: the preoperative polysomnography investigation had cumulated approximately the same 708 minutes, while the postoperative investigation cumulated 565 minutes; also, the total sleeping times are different in size - 334 minutes compared to 417 minutes during the second investigation. And this can be a factor which can change the statistic values.

The arousals index decreased from $28.8 / \mathrm{h}$ to 19.1/h, from a total number of events of 206 to 133 (Figure 9).

At 6 months postoperatively, the body mass index improved from 33.2 to 30.4 (no modification of the obesity category - grade I) and this can be one of the most important factors implicated in the modifications of the polysomnography values. As in the first case, one of the reasons for the differences between the two examination results can be the "first night effect" ${ }^{\prime 3436}$.

The overall effect in both patients showed a significant reduction in AHI score and REM sleep improvement and also a decreasing in the total number of arousals. Postoperative results show changes in sleep by decreasing the number of arousals and favouring the achievement of a higher index of REM sleep. A significant improvement in sleep apnea symptoms occurred in our patients. The polysomnogram parameters were improved after LAUP and septoplasty with mucotomy. Subjective symptoms were also improved after the surgery. In both cases, the arousal index improved and, as we know, arousals are represented by the ability to wake up upon external stimulation, and they enable an organism to react adequately to danger signals; also, rapid eye movement (REM) sleep is accompanied by several phenomena that increase the vulnerability and endangerment of an organism against external threat ${ }^{37,38}$. By decreasing the number of arousals, it changed the sleep architecture by reaching a higher REM sleep index in both patients.

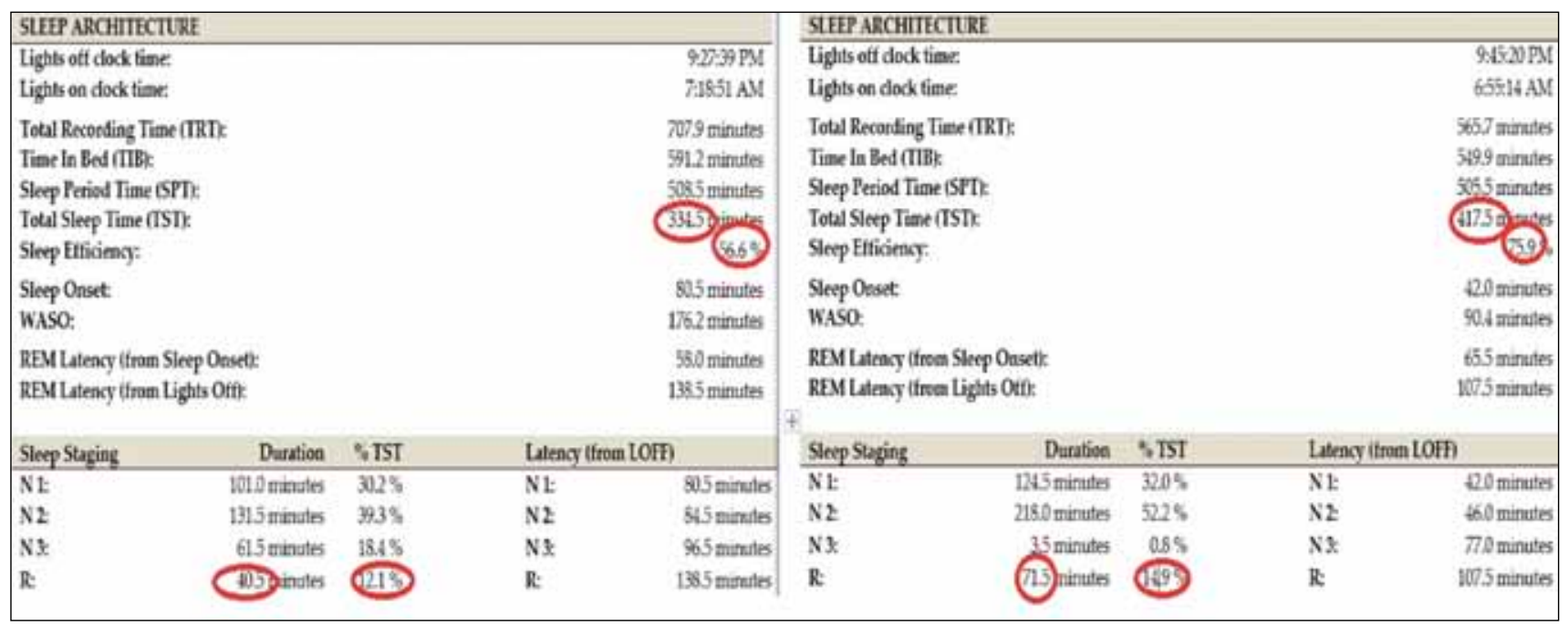

Figure 8 After septoplasty and mucotomy, the sleep efficiency was increased from $56.6 \%$ to $75.9 \%$ and REM sleep obtained a better result: 40.5 preoperatively and 71.5 minutes on 6 months postoperatively.

\begin{tabular}{|c|c|c|c|c|c|c|}
\hline \multicolumn{7}{|l|}{ IROUSAIS } \\
\hline & REM & NREM & Arousals & Awakenings & $A t+d w$ & $\begin{array}{l}\text { Ar+Ass } \\
\text { lader }\end{array}$ \\
\hline Respindary: & 0 & 64 & 67 & 38 & 165 & 14.7 \\
\hline Leg Morcuent: & 0 & 22 & 22 & 18 & 4) & 56 \\
\hline Snore: & 0 & 8 & 8 & 10 & 18 & 25 \\
\hline Spontaneous: & 0 & 20 & 24 & 19 & 6 & 6.0 \\
\hline Total: & 0 & 114 & 121 & 8 & 266 & 288 \\
\hline Arossal lndec & 00 & 159 & 169 & 11.9 & 65 & - \\
\hline
\end{tabular}

\begin{tabular}{|c|c|c|c|c|c|c|}
\hline \multicolumn{7}{|l|}{ AROLSAIS } \\
\hline & REM & NREM & Arousals & Awakening & $A r+A v$ & $\begin{array}{l}\frac{A r}{A}+A \hbar \\
\text { Indes }\end{array}$ \\
\hline Respiratory: & 12 & 7 & 19 & 10 & 2 & 42 \\
\hline Leg Movenent: & 3 & 22 & 86 & 8 & 34 & 49 \\
\hline Sabre: & 0 & 1 & 1 & 3 & 4 & 0.6 \\
\hline Sposthaeous: & 9 & 18 & $\bar{x}$ & 36 & 66 & 95 \\
\hline Tots & 24 & 45 & 74 & 58 & 133 & 19.1 \\
\hline Anousal lindec & 15.7 & 8.8 & 106 & 85 & (191) & - \\
\hline
\end{tabular}

Figure 9 After nasal permeabilization surgery ( 6 months postoperatively), the arousals index decreased from a total of $28.8 / \mathrm{h}$ to a better score of 19.1 events per hour. 
After both kinds of permeabilization of the upper airway tract were performed, the quality of life on the Calgary score questionnaire has improved. The daytime sleepiness is no longer a hindrance in the patient's daily schedule.

\section{CONCLUSIONS}

The goal of permeabilization surgery of the upper airways tract is to have a normal respiration during sleep and to decrease the number and the intensity of snoring episodes.

Correlating the results obtained by different authors with the one obtained by us, we can conclude that permeabilization surgery, together with weight loss, have a positive effect on the quality of sleep and also on sleep distribution. Moreover, we can invoke the "first night" effect on the accuracy of the polysomnography investigation regarding the difference between preoperative and postoperative REM sleep time.

Our partial results showed, 6 months after the permeabilization surgery of the upper airway tract, that the arousals index decreased and sleep architecture underwent changes that consisted in decreasing the Stage 1 and Stage 2 sleep; therefore, REM sleep reached a better score.

Also, LAUP and nasal permeabilization surgery are generally well tolerated by the patients. The most important side effects are pharyngeal pain and sometimes velar insufficiency.

Conflict of interest: The authors have no conflict of interest.

Contribution of authors: All authors have equally contributed to this work.

\section{REFERENCES}

1. Benca R.M., Obermeyer W.H., Thisted R.A., Gillin J.C. - Sleep and psychiatric disorders. A meta-analysis. Arch Gen Psychiatry, 1992;49(8):651668; discussion 669-670.

2. Agnew H.W., Webb W.B. - Measurement of sleep onset by EEG criteria. Am J EEG Technol., 1972;12(3):127-134.

3. Czeisler C.A., Zimmerman J.C., Ronda J.M., Moore-Ede M.C., Weitzman E.D. - Timing of REM sleep is coupled to the circadian rhythm of body temperature in man. Sleep, 1980;2(3):329-346.

4. Zulley J. - Distribution of REM sleep in entrained 24 hour and free-running sleep-wake cycles. Sleep, 1980;2(4):377-389.

5. Busby K., Pivik R.T. - Failure of high intensity auditory stimuli to affect behavioral arousal in children during the first sleep cycle. Pediatr Res., 1983;17(10):802-805.

6. Agnew H.W., Webb W.B., Williams R.L. - The first-night effect: an EEG study of sleep. Psychophysiology, 1966;2(3):263-266.

7. Parmeggiani P.L. - Temperature regulation during sleep: a study in homeostasis. In: Orem J., Barnes C.D., ed. Physiology in sleep. Research
Topics in Physiology. New York: Academic Press, 1980;p.98-143.

8. Freemon F.R. - The effect of chronically administered delta-9-tetrahydrocannabinol upon the polygraphically monitored sleep of normal volunteers. Drug Alcohol Depend., 1982;10(4):345-353.

9. Rupp T.L., Acebo C., Van Reen E., Carskadon M.A. - Effects of a moderate evening dose of alcohol. I: Sleepiness. Alcohol Clin Exp Res., 2007;31(8):1358-1364. Epub 2007 Jun 5.

10. Jacobowitz O. - Palatal and tongue base surgery for surgical treatment of obstructive sleep apnea: a prospective study. Otolaryngol Head Neck Surg., 2006;135(2):258-264.

11. Chisholm E., Kotecha B. - Oropharyngeal surgery for obstructive sleep apnoea in CPAP failures. Eur Arch Otorhinolaryngol., 2007;264(1):5155. Epub 2006 Aug 31.

12. Ferguson K.A., Heighway K., Ruby R.R. - A randomized trial of laser-assisted uvulopalatoplasty in the treatment of mild obstructive sleep apnea. Am J Respir Crit Care Med., 2003;167(1):15-19.

13. Verse T., Maurer J.T., Pirsig W. - Effect of nasal surgery on sleep-related breathing disorders. Laryngoscope, 2002;112(1):64-68.

14. Koutserelakis I., Georgoulopoulos G., Perraki E., Vagiakis E., Roussos C., Zakynthinos S.G. - Randomised trial of nasal surgery for fixed nasal obstruction in obstructive sleep apnea. Eur Respir J., 2008;31(1):110-117. Epub 2007 Sep 26.

15. Papsidero M.J. - The role of nasal obstruction in obstructive sleep apnea syndrome. Ear Nose Throat J., 1993;72(1):82-84.

16. Rosow D.E., Stewart M.G. - Is nasal surgery an effective treatment for obstructive sleep apnea? Laryngoscope, 2010;120:1496-1497.

17. Olsen K.D., Kern E.B. - Nasal influences on snoring and obstructive sleep apnea. Mayo Clin Proc., 1990;65(8):1095-1105.

18. Dayal V.S., Phillipson E.A. - Nasal surgery in the management of sleep apnea. Ann Otol Rhinol Laryngol., 1985;94(6 Pt 1):550-554.

19. Friedman M., Tanyeri H., Lim J.W., Landsberg R., Vaidyanathan K., Caldarelli D. - Effect of improved nasal breathing on obstructive sleep apnea. Otolaryngol Head Neck Surg., 2000;122(1):71-74

20. Li H.Y., Lin Y., Chen N.H., Lee L.A., Fang T.J., Wang P.C. - Improvement in quality of life after nasal surgery alone for patients with obstructive sleep apnea and nasal obstruction. Arch Otolaryngol Head Neck Surg., 2008;134(4):429-433. doi: 10.1001/archotol.134.4.429.

21. Jiten N., Ramalingam W.V.B.S., Rajput A.K., Giriraj Singh, Mukesh Chandra, kalpana Th., Satish N. - Laser-Assisted Uvulopalatoplasty for Management of Obstructive Sleep Apnoea: Pre and Post Operative Analysis Using Parameters of Polysomnography and Magnetic Resonance Imaging. IOSR-JDMS, 2015;14(8ver.II):105-111.

22. Kamami Y.V. - Laser CO2 for snoring - preliminary results. Acta Otorhinolaryngol Belg., 1990;44(4):451-456.

23. Mickelson S.A. - Laser-assisted uvulopalatoplasty for obstructive sleep apnea. Laryngoscope, 1996;106(1):10-13.

24. Walker R.P., Grigg-Damberger M.M., Gopalsami C. Uvulopalatopharyngoplasty versus laser-assisted uvulopalatoplasty for the treatment of obstructive sleep apnea. Laryngoscope, 1997;107(1):76-82.

25. Lysdahl M., Haraldsson P.O. - Uvulopalatopharyngoplasty versus laser uvulopalatoplasty: prospective long-term follow-up of self-reported symptoms. Acta Otolaryngol., 2002;122(7):752-757.

26. Lin C.C., Lee K.S., Chang K.C., Wu K.M., Chou C.S. - Effect of laser-assisted uvulopalatoplasty on oral airway resistance during wakefulness in obstructive sleep apnea syndrome. Eur Arch Otorhinolaryngol., 2006;263(3):241-247. doi: 10.1007/s00405-005-0994-2.

27. Ryan C.F., Love L.L. - Unpredictable results of laser assisted uvulopalatoplasty in the treatment of obstructive sleep apnoea. Thorax, 2000;55(5):399-404

28. Carenfelt C. - Laser uvulopalatoplasty in treatment of habitual snoring Ann Otol Rhinol Laryngol., 1991;100:451-454. 
29. Barnes M., Houston D., Worsnop C.J., Neill A.M., Mykytyn I.J., Kay A., Trinder J., Saunders N.A., Douglas McEvoy R., Pierce R.J. - A randomized controlled trial of continuous positive airway pressure in mild obstructive sleep apnea. Am J Respir Crit Care Med., 2002;165(6):773-780.

30. Giles T.L, Lasserson T., Smith B., White J., Wright J., Cates C.J. Continuous positive airways pressure for obstructive sleep apnoea in adults. Cochrane Database Syst Rev., 2006;(1):CD001106.

31. Berry R.B., Budhiraja R., Gottlieb D.J., Gozal D., Iber C., Kapur V.K., Marcus C.L., Mehra R., Parthasarathy S., Quan S.F., Redline S., Strohl K.P., Davidson Ward S.L., Tangredi M.M.; American Academy of Sleep Medicine. - Rules for scoring respiratory events in sleep: update of the 2007 AASM Manual for the Scoring of Sleep and Associated Events. Deliberations of the Sleep Apnea Definitions Task Force of the American Academy of Sleep Medicine. J Clin Sleep Med., 2012;8(5):597-619. doi: $10.5664 /$ jc.sm.2172.

32. Bonnet M.H., Carley D.W., Carskadon M.A., Easton P.A., et al. - EEG arousals: scoring rules and examples. A preliminary report from the Sleep Disorders Atlas Task Force of the American Sleep Disorder
Association. Sleep, 1992;15(2):174-184.

33. Guyatt G.H., Feeny D.H., Patrick D.L. - Measuring health-related quality of life. Ann Intern Med., 1993;118(8):622-629.

34. Tamaki M., Nittono H., Hayashi M., Hori T. - Examination of the firstnight effect during the sleep-onset period. Sleep, 2005;28(2):195-202.

35. Bon O.L., Minner P., Van Moorsel C., Hoffmann G., Gallego S., Lambrecht L., Pelc I., Linkowski P. - First-night effect in the chronic fatigue syndrome. Psychiatry Res., 2003;120(2):191-199.

36. Moser D., Kloesch G., Fischmeister F.P., Bauer H., Zeitlhofer J. - Cyclic alternating pattern and sleep quality in healthy subjects - Is there a firstnight effect on different approaches of sleep quality? Biological Psychology, 2010;83(1):20-26.

37. Lindberg E., Gislason T. - Epidemiology of sleep-related obstructive breathing. Sleep Med Rev., 2000;4(5):411-433.

38. Young T., Peppard P.E. - Epidemiology of obstructive sleep apnea. In: McNicholas W.T., Phillipson E.A., editors. - Breathing disorders in sleep. London: W. B. Saunders, 2002;p.31-43. 